\title{
Degree based and neighbourhood degree-sum based topological indices of PAH(Dimer 1) in graphene context
}

\author{
Tamilarasi.C ${ }^{\text {a }}$, F. Simon Raj ${ }^{\text {b }}$ \\ ${ }^{\mathrm{a}, \mathrm{b}}$ Hindustan Institute of Technology and Science, Chennai, Tamilnadu, India.
}

Article History: Received: 10 November 2020; Revised 12 January 2021 Accepted: 27 January 2021; Published online: 5 April 2021

\begin{abstract}
In this paper, twenty degree-based topological indices and seven neighbourhood degree-sum-based topological indices of Dimer 1 (two units of chrysene) [4] 0D \& 1D in the graphene context are enumerated. The Oligomer Approach[3] is practiced here to explore the interconnection between PAH ( cove type periphery based on 11, 11'-dibromo-5,5'-bis chrysene as a key monomer-Dimer 1) and graphene numerically through the indices.

Keywords: Neighbourhood degree, Degree of vertex, TIs (Topological Indices), NTIs (Neighbourhood Topological Indices), Oligomer Approach, cove-edged, (11, 11'-dibromo-5,5'-bischrysene), Dimer 1, Linear Chain(LCn), Radial Expansion, PAHs (Polycyclic Aromatic Hydrocarbons), GQDs (Graphene Quantum Dots), GNRs (Graphene Nano Ribbons), GNS (Graphene Nano Sheets)
\end{abstract}

\section{Introduction}

The separation of the Graphene (21st-century wonder material) layer from graphite is the recent revolution in the material science domain. Polycyclic aromatic hydrocarbons $(P A H \mathrm{~s}$ or polyaromatic hydrocarbons or polynuclear aromatic hydrocarbons) are organic compounds having only carbon and hydrogen with the collection of multiple aromatic rings. In this paper, Dimer $1(P A H)$ is considered to evaluate in the graphene context. Graphene monomer $(G Q D, 0 D)$ is quasi-zero-dimensional nanographene cut-out. Graphene nanoribbon $(G N R, 1 D)$ is quasi-one-dimensional (variation in one direction only) graphene cut out and Graphene nanosheet $(G N S, 2 D)$ is quasi-two-dimensional graphene cut-out. "Oligomer approach" consists of few repeating finite units of monomers. In this paper, the focus on $P A H \mathrm{~s}$ in "graphene context" resulting in $0 D(G Q D), 1 D(G N R)$ molecular structureproperty related Topological index development.

Chemical graph theory $(C G T)$ is the division of mathematical Chemistry and graph theory is used as the mathematical model of molecular structures to predict the physical properties of the molecules. Abundant studies specify that the relation between the chemical properties of the compounds and their molecular structures are significantly related. Topological indices demarcated on these molecular structures to predict the physicochemical properties and biological activity. Topological indices are engendered as new limits in quantitative structureproperty relationship ( QSPR/QSAR) models to encrypt the structural environment of each atom in a molecule. The two main branches of topological indices are distance-based and degree-based. The degree-based Topological indices (TIs) are stretched to neighbourhood degree sum topological indices (NTIs). The neighbourhood degree sum of a vertex $\mathrm{v}$ is the sum of the degrees of neighbourhood vertices of the vertex $v$ and is denoted as $S_{v}$, whereas the degree of $v$ is the number of edges meeting at $\mathrm{v}$ and is denoted as $d_{v}$ Chemical graph theory $(C G T)$ is a division of mathematical chemistry, pacts with the nontrivial applications of graph theory to crack molecular problems. At large, a molecular graph is used to characterize molecules by seeing the atoms as the vertices of the graph and the molecular bonds as the edges. CGT is to custom algebraic invariants to condense the topological structure of a molecule to a single number, which symbolizeseither the energy of the molecule as a whole or its orbitals. 

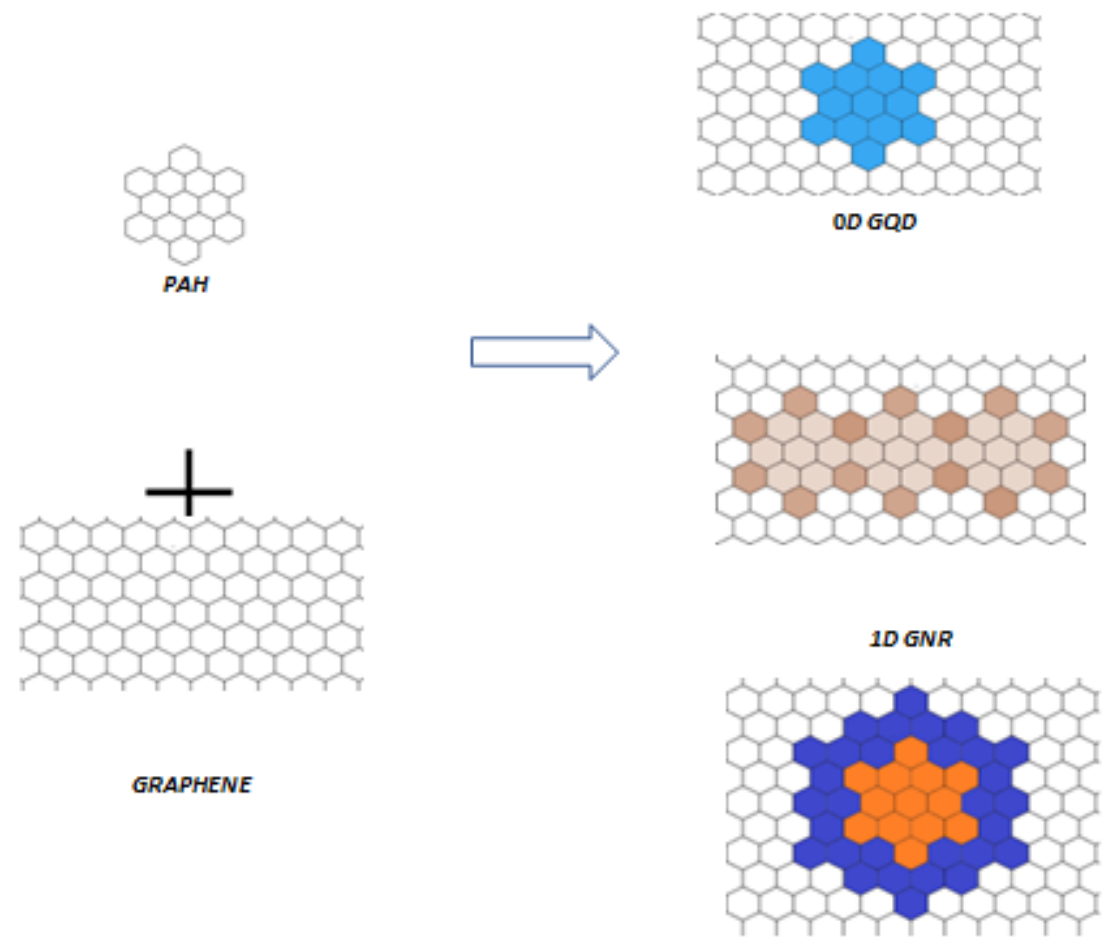

$2 D$ GNS

Figure 1. Example of diagrammatical representation of $P A H \mathrm{~s}$ in "graphene context" describing $0 D(G Q D), 1 D$ $(G N R), 2 D(G N S)$ molecular structures respectively.

\section{Preliminaries}

Let $D($ Dimer 1$)$ denotes a molecular graph of a distinct unit of cove type periphery based on 11, 11'-dibromo5,5'-bis chrysene as a key monomer-dimer 1 ( 0 D oligomers). Let $L C_{n} \quad$ (Dimer 1$)$ denotes a molecular graph of a linear chain of n number of monomers of Dimer 1 where the chemical structure is treated as a single unit and $n$ number of units ( $1 \mathrm{D}$ oligomers) are linearly arranged like a ribbon. We determined here, GNR containing $\mathrm{n}$ number of subunits of Dimer 1as Linear Chain of Dimer 1 ( $L C n)$

Let $\mathrm{N}(V, E)$ is a simple, connected graph where $V(\mathrm{~N})$ is its vertex set and $E(\mathrm{~N})$ is its edge set for any vertex $\kappa \epsilon$ $V(\mathrm{~N})$ and for any edge $\kappa, \vartheta \in E(\mathrm{~N})$. The degree of vertex $\kappa \in V$ denotes as $(\kappa)$ and the neighbourhood degree sum of $\kappa \in V$ denotes as $S(\kappa)$

Here, we have tabulated the twenty degree-based topological indices and seven neighbourhood degree-based topological indices along with their notations and descriptions. Based on the listed TIs and NTIs, D $($ Dimer 1$), L C_{n}$ (Dimer 1) are enumerated.

Table $1 \& 2$. The notations and Descriptions of twenty TIs and seven NTIs are listed here.

Table1.

\begin{tabular}{|l|l|}
\hline Topological Indices - TIs & Notations \& Descriptions \\
\hline [8] Randic Index & $R(\mathrm{~N})=\sum_{\kappa, \vartheta \in E} \frac{1}{\sqrt{\eta(\kappa) \eta(\vartheta)}}$ \\
\hline [8] Generalise ${ }_{\mathrm{d}}$ Randić Index & $R_{\boldsymbol{\alpha}}(\mathrm{N})=\sum_{\kappa, \vartheta \in E}(\eta(\kappa) \eta(\vartheta))^{\alpha}$ \\
\hline [11] Reciprocal Randić Index & $R R(\mathrm{~N})=\sum_{\kappa, \vartheta \in E} \sqrt{(\eta(\kappa) \eta(\vartheta))}$ \\
\hline [11] Reduced Reciprocal Randić Index & $R R R(\mathrm{~N})=\sum_{\kappa, \vartheta \in E} \sqrt{(\eta(\kappa)-1)(\eta(\vartheta)-1)}$ \\
\hline
\end{tabular}




\begin{tabular}{|c|c|}
\hline [12] Sum Connectivity Index & $S(\mathrm{~N})=\sum_{\kappa, \vartheta \in E} \frac{1}{\sqrt{\eta(\kappa)+\eta(\vartheta)}}$ \\
\hline [6] First Zagreb Index & $\begin{array}{l}M_{1}(\mathrm{~N})=\sum_{\kappa \in V} \eta(\kappa)^{2} \text { or } \\
M_{1}(\mathrm{~N})=\sum_{\kappa, \vartheta \in E} \eta(\kappa)+\eta(\vartheta)\end{array}$ \\
\hline [6] Second Zagreb Index & $M_{2}(\mathrm{~N})=\sum_{\kappa, \vartheta \in E} \eta(\kappa) \eta(\vartheta)$ \\
\hline [7] Third Zagreb Index & $M_{3}(\mathrm{~N})=\sum_{\kappa, \vartheta \in E}|\eta(\kappa)-\eta(\vartheta)|$ \\
\hline [5] Reclassified Zagreb Index 1 & $\operatorname{Re} Z_{1}(\mathrm{~N})=\sum_{\kappa, \vartheta \in E} \frac{\eta(\kappa) \eta(\vartheta)}{\eta(\kappa)+\eta(\vartheta)}$ \\
\hline [5] Reclassified Zagreb Index 2 & $\operatorname{Re}_{2}(\mathrm{~N})=\sum_{\kappa, \vartheta \in E} \frac{\eta(\kappa)+\eta(\vartheta)}{\eta(\kappa) \eta(\vartheta)}$ \\
\hline [5] Reclassified Zagreb Index 3 & $\operatorname{ReZ}_{3}(\mathrm{~N})=\sum_{\kappa, \vartheta \in E}(\eta(\kappa)+\eta(\vartheta))(\eta(\kappa) \eta(\vartheta))$ \\
\hline [11] Reduced Second Zagreb Index & $R M_{2}(N)=\sum_{\kappa, \vartheta \in E}(\eta(\kappa)-1)(\eta(\vartheta)-1)$ \\
\hline [14] Hyper Zagreb Index 1 & $H M_{1}(N)=\sum_{\kappa, \vartheta \in E}(\eta(\kappa)+\eta(\vartheta))^{2}$ \\
\hline [14] Hyper Zagreb Index 2 & $H M_{2}(N)=\sum_{\kappa, \vartheta \in E}(\eta(\kappa) \eta(\vartheta))^{2}$ \\
\hline [16] Augmented Zagreb Index & $\mathrm{AZ}(\mathrm{N})=\sum_{\kappa, \vartheta \in E}\left(\frac{\eta(\kappa) \eta(\vartheta)}{\eta(\kappa)+\eta(\vartheta)-2}\right)^{3}$ \\
\hline [15] Harmonic Index & $H(\mathcal{N})=\sum_{\kappa, \vartheta} \in E \frac{2}{(\eta(\kappa)+\eta(\vartheta))}$ \\
\hline [2] Atom Bond Connectivity Index & $A B C(N)=\sum_{\kappa, \vartheta \in E}\left(\sqrt{\frac{(\eta(\kappa)+\eta(\vartheta))-2}{(\eta(\kappa) \eta(\vartheta))}}\right)$ \\
\hline [3] Geometric Arithmetic Index & $G A(N)=\sum_{\kappa, \vartheta \in E}\left(\frac{2 \sqrt{(\eta(\kappa) \eta(\vartheta))}}{(\eta(\kappa)+\eta(\vartheta))}\right)$ \\
\hline [28] Forgotten Index & $F(N)=\sum_{\kappa \in V}(\eta(\kappa))^{3}$ or $\sum_{\kappa, \vartheta \in E} \eta(\kappa)^{2}+\eta(\vartheta)^{2}$ \\
\hline [14] Symmetric Division Index & $\begin{array}{l}S D D(N)=\sum_{\kappa, \vartheta \in E}\left(\left(\frac{\max ((\eta(\kappa), \eta(\vartheta))}{\min ((\eta(\kappa), \eta(\vartheta))}+\right.\right. \\
\left.\left.\frac{\min ((\eta(\kappa), \eta(\vartheta))}{\max ((\eta(\kappa), \eta(\vartheta))}\right)\right)\end{array}$ \\
\hline
\end{tabular}

Table 2.

\begin{tabular}{|l|l|}
\hline Neighbourhood Topological Indices & Notations \& Descriptions \\
\hline [40] Fourth Atom Bond Connectivity Index & $A B C_{4}(N)=\sum_{\kappa, \vartheta \in E} \sqrt{\frac{S(\kappa)+S(\vartheta)-2}{S(\kappa) S(\vartheta)}}$ \\
\hline [40] Fifth Geometric Arithmetic Index & $G A_{5}(N)=\sum_{\kappa, \vartheta \in E} \frac{2 \sqrt{S(\kappa) S(\vartheta)}}{S(\kappa)+S(\vartheta)}$ \\
\hline [32,33] Sanskruti Index & $S K(N)=\sum_{\kappa, \vartheta \in E}\left(\frac{S(\kappa) S(\vartheta)}{S(\kappa)+S(\vartheta)-2}\right)^{3}$ \\
\hline [14] Neighbourhood Version Of Forgotten Index & $F_{N}(N)=\sum_{\kappa \in V} S(\kappa)^{3}$ \\
\hline $\begin{array}{l}\text { [14] Modified Neighbourhood Version Of Forgotten } \\
\text { Index }\end{array}$ & $F_{N}{ }^{*}(N)=\sum_{\kappa, \vartheta \in E}\left(S(\kappa)^{2}+S(\kappa)^{2}\right)$ \\
\hline [14] Neighbourhood Version Of Second Zagreb Index & $M_{2}{ }^{*}(N)=\sum_{\kappa, \vartheta \in E} S(\kappa) S(\vartheta)$ \\
\hline [14] Neighbourhood Version Of Hyper Zagreb Index & $H M_{N}(N)=\sum_{\kappa, \vartheta \in E}(S(\kappa)+S(\vartheta))^{2}$ \\
\hline
\end{tabular}




\section{Methods and Main Results}

In this section, twenty degree-based topological indices and seven degree-sum-based topological indices mentioned in Table $1 \& 2$ are computed in 3 divisions based on $0 D, 1 D \& 2 D$ oligomers. For getting the results, we have employed vertex partition, edge partition, vertex partition of neighbourhood degree sum $\&$ edge partition of neighbourhood degree sum and are displayed in the Tables 3,4,5\& 6

The results of Distinct unit ( $0 \mathrm{D}$ approach) \& Linear Chain ( $1 D$ approach) at $n$ number of molecular graphs of the cove type periphery based on 11,11'-dibromo-5,5'-bis chrysene as a key monomer-Dimer 1 are presented in an elaborated manner.

Table $3 \& 4$. Vertex partition and neighbourhood degree sum partition of each vertex of $L C_{n}($ Dimer 1$)$, based on the degree of end vertices of each edge.

Table $5 \& 6$. Edge partition based on degrees of end vertices and degree sum of neighbours of end vertices of $L C_{n}$.

Table 3.

Table 4.

Table 5.

Table 6.

\begin{tabular}{|c|c|c|c|c|c|}
\hline \multirow{2}{*}{$\left|V\left(L C_{n}\right)\right|$} & \multirow{2}{*}{ Cardinality } & $\left|S\left(L C_{n}\right)\right|$ & Cardinality & $\left|E_{(\kappa, \vartheta)}\right|$ & Cardinality \\
\hline & & S I & $4 n+2$ & $\mid E_{0}$, & $8 n+4$ \\
\hline \multirow[t]{2}{*}{$\left|V_{2}\right|$} & \multirow[t]{2}{*}{$12 n+6$} & $\left|\mathrm{~S}_{4}\right|$ & $4 n+2$ & & \\
\hline & & $\left|S_{5}\right|$ & $8 n+4$ & $\left|E_{(2,3)}\right|$ & $8 n+4$ \\
\hline$\left|V_{3}\right|$ & $24 n-6$ & $\left|S_{7}\right|$ & 2 & $\left|E_{(3,3)}\right|$ & $32 n-11$ \\
\hline \multirow{3}{*}{$|V|$} & $30 n$ & $\left|S_{8}\right|$ & $8 n$ & $\begin{array}{l}\text { No. Of } \\
\text { Edges }\end{array}$ & $48 n-3$ \\
\hline & & $\left|S_{9}\right|$ & $16 n-8$ & & \\
\hline & & $\left|S_{\kappa}\right|$ & $36 n$ & & \\
\hline
\end{tabular}

\begin{tabular}{|l|l|}
\hline$\left|S_{(\kappa, 9)}\right|$ & Cardinality \\
\hline$\left|S_{(4,4)}\right|$ & 2 \\
\hline$\left|S_{(4,5)}\right|$ & $8 n$ \\
\hline$\left|S_{(5,5)}\right|$ & 2 \\
\hline$\left|S_{(5,7)}\right|$ & 4 \\
\hline$\left|S_{(5,8)}\right|$ & $8 n$ \\
\hline$\left|S_{(8,8)}\right|$ & 4 \\
\hline$\left|S_{(8,9)}\right|$ & $16 n-8$ \\
\hline$\left|S_{(7,9)}\right|$ & 2 \\
\hline$\left|S_{(9,9)}\right|$ & $16 n-9$ \\
\hline $\begin{array}{l}\text { No. of } \\
\text { edges }\end{array}$ & $48 n-3$ \\
\hline
\end{tabular}
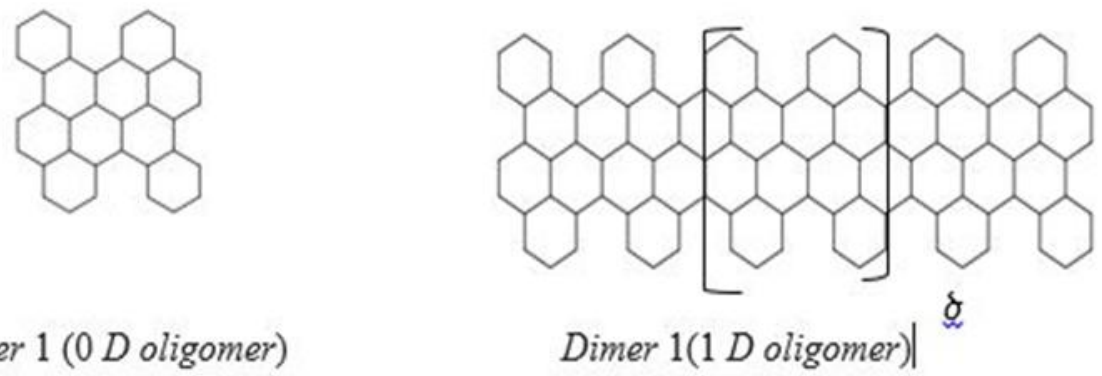

Dimer 1 (0 D oligomer)

Figure $2 \& 3$ show the monomer of cove type periphery based on $11,11^{\prime}$-dibromo-5,5' - bis chrysene $(0 D)$ and the Linear Chain at $\delta$ number of the monomers $(1 D)$.

Theorem 1.1: The Randić Indices of Linear Chain of (Dimer 1) is given by

(i) $R\left(L C_{n}\right)=17.93266 n-0.03368$

(ii) $R_{\boldsymbol{\alpha}}\left(L C_{n}\right)=(8 n+4) 4^{\alpha}+(8 n+4) 6^{\alpha}+(32 n-11) 9^{\alpha}$

(iii) $R R\left(L C_{n}\right)=131.59592 n-15.20204$

(iv) $\operatorname{RRR}\left(L C_{n}\right)=83.3137 n-12.34315$

Proof: Let $\kappa, \in E(L C n)$ and $(\kappa)$ and $\eta(\vartheta)$ are the degree of $\kappa$ and degree of $\vartheta$ respectively,

(i) $R\left(L C_{n}\right)=\frac{(8 n+4)}{2}+\frac{(8 n+4)}{\sqrt{6}}+\frac{(32 n-11)}{3}=17.93266 n-0.03368$

(ii) $R_{\alpha}\left(L C_{n}\right)=(8 n+4) 4^{\alpha}+(8 n+4) 6^{\alpha}+(32 n-11) 9^{\alpha}$ 
(iii)

$$
R R\left(L C_{n}\right)=2(8 n+4)+\sqrt{ } 6(8 n+4)+3(32 n-11)=131.59592 n-15.20204
$$

(iv)

$R R R\left(L C_{n}\right)=8 n+4+\sqrt{ } 2(8 n+4)+2(32 n-1)=83.3137 n-12.34315$

Theorem 1.2: The Sum Connectivity Index of Linear Chain of (Dimer 1) is given by

$S\left(L C_{n}\right)=20.64166 n-0.70188$

Proof: $\frac{(8 n+4)}{2}+\frac{(8 n+4)}{\sqrt{5}}+\frac{(32 n-11)}{\sqrt{6}}=20.64166 n-0.70188$

Theorem 1.3: The Zagreb Indices and their redefined version indices of Linear Chain of given by

(Dimer 1) is

(i) $\quad M_{1}\left(L C_{n}\right)=264 n-30$

(ii) $M_{2}\left(L C_{n}\right)=368 n-59$

(iii) $M_{3}\left(L C_{n}\right)=8 n+4$

(iv) $\operatorname{Re} Z_{1}\left(L C_{n}\right)=65.6 n-7.7$

(v) $\operatorname{Re} Z_{2}\left(L C_{n}\right)=36 n$

(vi) $\operatorname{ReZ}_{3}\left(L C_{n}\right)=2000 n-458$

(vii) $R M_{2}\left(L C_{n}\right)=152 n-32$

(viii) $H M_{1}\left(L C_{n}\right)=1480 n-232$

(ix) $H M_{2}\left(L C_{n}\right)=3008 n-683$

(x) $\quad A Z\left(L C_{n}\right)=492.39063 n-61.29688$

Proof: For getting the result of First Zagreb index, we can use the vertex partition and edge partition methods and other TIs were getting through edge partition method.

(i) (a) $M_{1}\left(L C_{n}\right)=4(12 n+6)+9(24 n-6)=264 n-30$

(b) $M_{1}\left(L C_{n}\right)=4(8 n+4)+5(8 n+4)+6(32 n-11)=264 n-30$

(ii) $M_{2}\left(L C_{n}\right)=4(8 n+4)+6(8 n+4)+9(32 n-11)=368 n-59$

(iii) $M_{3}\left(L C_{n}\right)=8 n+4$

(iv) $R e Z_{1}\left(L C_{n}\right)=(8 n+4)+\frac{6(8 n+4)}{5}+\frac{3(32 n-11)}{2}=65.6 n+7.7$

(v) $\operatorname{Re} Z_{2}\left(L C_{n}\right)=(8 n+4)+\frac{5(8 n+4)}{6}+\frac{2(32 n-11)}{3}=36 n$

(vi) $\operatorname{Re} Z_{3}\left(L C_{n}\right)=16(8 \mathrm{n}+4)+30(8 \mathrm{n}+4)+54(32-11)=2000 \mathrm{n}-458$

(vii) $R M_{2}\left(L C_{n}\right)=(8 n+4)+2(8 n+4)+4(32 n-11)=152 n-32$

(viii) $\mathrm{HM}_{1}\left(L C_{n}\right)=16(8 n+4)+25(8 n+4)+36(32 n-11)=1480 n-232$

(ix) $H M_{2}\left(L C_{n}\right)=16(8 n+4)+36(8 n+4)+81(32 n-11)=3008 n-683$

(x) $A Z\left(L C_{n}\right)=8(8 n+4)+8(8 n+4)+11.390625(32 n-11)=492.39063 n-61.29688$

Theorem 1.4: The Harmonic Index of Linear Chain is given by $H\left(L C_{n}\right)=17.86667 n-0.06667$

Proof: This index is calculated through edge partition method where $\kappa, \in E \& \kappa, \vartheta$ are the vertices of Linear chain of (Dimer 1).

$H\left(L C_{n}\right)=\frac{8 n+4}{2}+\frac{2(8 n+4)}{5}+\frac{(32 n-11)}{3}=17.86667 n-0.06667$

Theorem 1.5: The Atom Bond Connectivity Index of LCnis given by,

$A B C\left(L C_{n}\right)=32.64704 n-1.67648$

Proof: This index is calculated through edge partition method where $\kappa$ and $\vartheta$ are adjacent vertices of Linear Chain of Dimer 1. 
$A B C\left(L C_{n}\right)=\frac{(8 n+4)}{\sqrt{2}}+\frac{(8 n+4)}{\sqrt{2}}+\frac{2(32 n-11)}{3}=32.64704 n-1.67648$

Theorem 1.6: The Geometric Arithmetic Index of the Linear Chain of Dimer 1 is given by

$G A\left(L C_{n}\right)=37.1717 n+0.58585$

Proof: Here, we have used edge partition method for finding $G A\left(L C_{n}\right)$

$$
=(8 n+4)+\frac{2 \sqrt{6}}{5}(8 n+4)+(32 n-11)=37.1717 n+0.58585
$$

Theorem 1.7: The Forgotten Index of the linear chain is given by

$F\left(L C_{n}\right)=744 n-114$

Proof: Here, for getting the result of $F\left(L C_{n}\right)$, we can use vertex partition and edge partition methods.

(a) By vertex partition method, $\mathrm{u} \in V\left(L C_{n}\right)$

$F\left(L C_{n}\right)=8(12 n+6)+27(24 n-6)=744 n-114$

(b) By edge partition method, $\kappa, \vartheta \in E\left(L C_{n}\right)$

$F\left(L C_{n}\right)=8(8 n+4)+13(8 n+4)+18(32 n-11)=744 n-114$.

Theorem 1.8: The Symmetric Division Index of the Linear Chain is given by,

$S D D\left(L C_{n}\right)=97.33333 n-5.33333$

Proof: The result of $\operatorname{SDD}\left(L C_{n}\right)$ is done by edge partition method where $\kappa, \vartheta \in E\left(L C_{n}\right)$.

$S D D\left(L C_{n}\right)=2(8 n+4)+\left(\frac{13}{6}\right)(8 n+4)+2(32 n-11)=97.33333 n-5.33333$

Theorem 2.1: The fourth Atom Bond Connectivity Index of the Linear Chain of Dimer 1 is given by,

$A B C_{4}\left(L C_{n}\right)=23.34218 n-0.34365$

Proof: Here, we have calculated $A B C_{4}$ Index by using degree sum of neighbourhood method.

$A B C_{4}\left(L C_{n}\right)=2 \sqrt{\frac{6}{16}}+(8 n) \sqrt{\frac{7}{20}}+0.4 \sqrt{8}+4 \sqrt{\frac{10}{25}}+(8 n) \sqrt{\frac{11}{40}}+0.5 \sqrt{14}+\quad(16 n-8) \sqrt{\frac{15}{72}}+2 \sqrt{\frac{14}{63}}+(16 n-$ 9) $\frac{4}{9}=23.34218 n-0.34365$

Theorem 2.2: The fifth version of Geometric Arithmetic Index of $L C_{n}$ of is given by,

$G A_{5}\left(L C_{n}\right)=47.70682 n-3.05779$

Proof: Let $\kappa, \in E$ and the neighbourhood degree of $\kappa$ and $\vartheta$ are denoted as $S(\kappa)$ and $S(\vartheta)$ respectively. The procedure of getting the result is as follows:

$$
\begin{aligned}
G A_{5}\left(L C_{n}\right) & =2+(16 n) \frac{\sqrt{20}}{9}+2+\frac{8 \sqrt{35}}{12}+(16 n) \frac{\sqrt{40}}{13}+4+(32 n-16) \frac{\sqrt{72}}{17}+\frac{\sqrt{63}}{4}+(16 n-9) \\
& =47.70682 n-3.05779 .
\end{aligned}
$$

Theorem 2.3: The Sanskruti Index of linear chain is given by,

$S K\left(L C_{n}\right)=4416.67551 n-1217.60784$

Proof: The Sanskruti Index is calculated based on degree sum of neighbourhood method where $\kappa, \vartheta \in E\left(L C_{n}\right)$.

$$
\begin{aligned}
& S K\left(L C_{n}\right)=\sum_{u, v \in}\left(\frac{s_{u} \cdot s_{v}}{s_{u}+s_{v}-2}\right)^{3}=2\left(\frac{16}{6}\right)^{3}+(8 n)\left(\frac{20}{7}\right)^{3}+2(30.51757)+4(42.875)+\left(\frac{40}{11}\right)^{3}(8 n)+4(95.53352)+ \\
& \left(\frac{72}{15}\right)^{3}(16 n-8)+2\left(\frac{63}{14}\right)^{3}+\left(\frac{81}{16}\right)^{3}(16 n-9)=4416.67551 n-1217.60784 \text {. }
\end{aligned}
$$

Theorem 2.4: The neighbourhood versions of Forgotten Index, modified Forgotten Index, Second Zagreb Index \& First Hyper Zagreb Index are given by,

a) $\quad F_{N}\left(L C_{n}\right)=17016 n-4518$

b) $\quad F_{N}{ }^{*}\left(L C_{n}\right)=5952 n-1386$ 
c) $\quad M_{2}{ }^{*}\left(L C_{n}\right)=2928 n-701$

d) $\quad H M_{N}\left(L C_{n}\right)=11808 n-2788$

Similar methodology followed in calculating twenty TIs and seven NTIs of the molecular graph of Dimer1 $(0 D)$ andtheir results are presented in Tables $7 \& 8$.

Table 7. Twenty topological indices of molecular graph of Dimer 1(0D oligomer).

\begin{tabular}{|l|l|l|l|}
\hline$T I \mathrm{~s}$ & Results of Dimer $1(0 \mathrm{D})$ & & \\
\hline$R($ Dimer 1$)$ & 17.89898 & $S($ Dimer 1$)$ & 19.93977 \\
\hline$R^{\alpha}($ Dimer 1$)$ & $12\left(4^{\alpha}\right)+12\left(6^{\alpha}\right)+21\left(9^{\alpha}\right)$ & $M_{1}($ Dimer 1$)$ & 234 \\
\hline$R R($ Dimer 1$)$ & 116.39388 & $M_{2}($ Dimer 1$)$ & 228 \\
\hline$R R R($ Dimer 1$)$ & 70.97056 & $M_{3}($ Dimer 1$)$ & 12 \\
\hline$R e Z_{1}($ Dimer 1$)$ & 57.9 & $A Z($ Dimer 1$)$ & 431.20313 \\
\hline$R e Z_{2}($ Dimer 1$)$ & 36 & $H($ Dimer 1$)$ & 17.8 \\
\hline$R e Z_{3}($ Dimer 1$)$ & 1686 & $A B C($ Dimer 1$)$ & 30.97056 \\
\hline$R M_{2}($ Dimer 1$)$ & 120 & $G A($ Dimer 1$)$ & 33.9798 \\
\hline$H M_{1}$ (Dimer 1$)$ & 1248 & $F($ Dimer 1$)$ & 630 \\
\hline$H M_{2}$ (Dimer 1$)$ & 2325 & $S D D($ Dimer 1$)$ & 105 \\
\hline
\end{tabular}

Table 8. Seven neighbourhood degree sum of topological indices of Dimer 1 ( $0 D$ oligomer).

\begin{tabular}{|l|l|l|l|l|}
\hline$N T I s$ & Results & & \\
\hline$A B C_{4}($ Dimer 1$)$ & 23.18799 & $F_{N}{ }^{*}($ Dimer 1$)$ & 4566 \\
\hline$G A_{5}($ Dimer 1$)$ & 44.6490 & $M_{2}{ }^{*}($ Dimer 1$)$ & 2227 \\
\hline$S K($ Dimer 1$)$ & 3199.06767 & $H M_{N}($ Dimer 1$)$ & 9020 \\
\hline$F_{N}($ Dimer 1$)$ & 12498 & & \\
\hline
\end{tabular}

\section{Conclusion}

Twenty numbers degree-based and seven numbers neighborhood degree-based topological indices are computed on the molecular graph of cove type periphery based on 11, 11'-dibromo-5,5'-bis chrysene as a key monomer-dimer 1 for $0 D \& 1 D$ monomers in graphene context. With the help of computed above topological indices, the outstanding properties of Dimer 1 in graphene context $(0 D \& 1 D)$ can be estimated for future new material developments thus helping the engineering industries especially semi-conductors.

This study will open up many new areas to explore all $P A H$ materials consideration in the graphene context to explore the future graphene era.

\section{References}

Wiener. H, Structural determination of paraffin boiling points. J. Am. Chem. Soc. 1947,69,17-20.

Randic, M. Characterization of molecular branching. J. Am. Chem. Soc. 1975,97,6609-6615.

Xiao- Ye Wang; XuelinYaq, Poly aromatic hydrocarbons in the graphene era, Sci China Chem, 2019, 62, 11426019-94.

Liu J, Li BW, Tan YZ, Giannakopoulos A, Sanchez-Sanchez C, Beljonne D, Ruffieux P, Fasel R, Feng X, Mullen K. J Am Chem Soc, 2015, 137: 6097-6103.

Klarner, D.A.; Polyominoes, S. W. In Handbook of Discrete and Computational Geometry, Goodman, J.E., O Rourke, J., Eds.; CRC Press, Boca Raton, 1997, pp.225-242.

Estrada, E.; Torres, 1.; Rodriguez, L., Gutman, I. An atom-bond connectivity index: Modelling the enthalpy of formation of alkanes. NISCAIR-CSIR 1998, 37A, 849-855.

West, D.B. Introduction to graph theory; Prentice-Hall: Upper Saddle River, NJ, USA, 2001; Volume 2.

Randic, M. The connectivity index 25 years after. J. Mol, Gr. Model. 2001, 20, 19-35 [CrossRef]

Gutman, L.; Furtula, B. Recent Results in the theory of Randic Index; University of Kragujevac and the Faculty of Science Kragujevac: Kragujevac, Serbia, 2008.

Li, X.; Shi, Y.A survey on the Randic Index. Match Commun. Math. Comput. Chem. 2008, 59,127-156.

M.H. Khalifeh; H. Yousefi- Azari; A.R. Ashrafi, The first and second Zagreb indices of some graph operations. Dis. App. Math. 157, 2009, 804-811.

Ghorbani,M.; Ghazi, M. Computing some topological indices of triangular benzenoid. Digest J. Nanomater. Biostruct. 2010, 5(4), 1107-11. 
L. Xiao, S. Chen, Z. Guo, Q. Chen, The geometric-arithmetic index of benzenoid systems and phenylenes, Int. J. Contemp. Math. Sci.,5,2010, 2225-2230.

SouravMondal; Nilanjan De, On Some New Neighbourhood Degree Based Indices, arXiv:1906.11215, physics.chem-ph, 2019.

L. Zhong, "The harmonic index for graphs", Applied Mathematics Letters, vol.25, no.3, pp.561-566,2012.

Yufei Huang; Bolian Liu; Lu Gan, Augumented Zagreb Index of Connected Graphs, Math. Comput. Chem. 67, 2012, 483-494.

L. Zhong, The harmonic index for graphs, Applied Mathematics Letters, 25, 2012, 561-566.

BharatiRajan; Albert William, On Certain Topological Indices of Silicate, Honeycomb and Hexagonal Networks, J.Comp. \& Math. Sci. Vol.3(5), 530-535, 2012.

Ivan Gutman, "Degree-Based Topological Indices”, Croat. Chem. Acta 86(4), 2013, 351-361.

Ghorbani, M; Hosseinzadeh, M.A. The third version of Zagreb index. Discret. Math. Algorithm. Appl. 2013, 5, article 1350039.

P.S. Ranjini, V. Lokesha, A. Usha, Relation between phenylene and hexagonal squeeze using harmonic index, Int. J. Graph Theory, 1, 2013, no. 4, 116-121.

J. X.Liu, "On harmonic index and diameter of graphs", Journal of Applied Mathematics and Physics, vol. I, no.3, pp. 5-6,2013.

G.H. Shirdel, H. Rezapour, and A.M. Sayadi, “The hyper Zagreb Index of graph operations”, Iranian Journal of Mathematical Chemistry, vol.no.2, pp.213-220,2013.

Farahani, M.R.; Vlad, M.P. Computing first and second Zagreb index, first and second Zagreb polynomial of capra-designed planar benzenoid series Cak(C6). StudiaUbbChemia. 2013, LVIII(2), 133-142.

Ivan Gutman; Boris Furtula; Clive Elphick. Three New/Old Vertex-Degree-Based Topological Indices, MATCH Commun. Math. Comput. Chem. 72,2014, 617-632

Yan, Li; Gao, W.; Li, J. General harmonic index and general sum connectivity index of polyomino chains and nanotubes. J. Comput. Theor. Nanosci. 2015, 12(10), 3940-3944.

Futula, B.; Gutman, I.; Vukicevic, Z.K.; Lekishvili, G.; Popivoda, G. On an old/new degree - based topological index, Bull., Cl. Sci. Math. Nat., Sci. 2015, 40, 19-31.

Futula, B.; Gutman, I. A forgotten topological index. J. Math. Chem. 2015, 53(4), 1184-90.

Kindar Das; KexiangXu, “Zagreb Indices of graphs” Front. Math. China., 2015, 0431-9.

Wei Gao; Weifan Wang; Mohammad Reza Farahani, "Topological Indices study of Molecular Structure in Anticancer Drugs". J. Chem. 2016, 321-327

Gao, W.; Farahani, M.R. Degree-based indices computation for special chemical molecular structures using edge dividing method. Appl. Math. Nonlinear Sci. 2016, 1(1), 94-117.

Shigehalli V. S.; Kanabur, R. Computation of new degree-based topological indices of graphene. J. Math. 2016, Article ID 4341919.

Muhammad ShoaibSardar; Xiang-Febg Pan, Computing Sanskruti Index of Titania Nanotubes, Open J. Math. Sci., Vol. 1, 2017, No. 1, pp. 126-131.

Hosamani, S. M. . Computing Sanskruti index of certain nanostructures. Journal of Applied Mathematics and Computing, 54(1-2), 2017, 425-433.

Sardar, M. S., Zafar, S., \& Farahani, M. R. Computing Sanskruti index of the Polycyclic Aromatic Hydrocarbons. Geology, Ecology, and Landscapes, 2017, 1(1), 37-40.

Abdul JahilManshadKhalaf; HaneenKareenAljanabiy, Computing the Harmonic Index for Alkanes, Alkenes and Alkynes, J. Eng. App. Sci.13, 2018, 2266-2273.

Imran, M.; Ali, M. A.; Ahmad, S.; Siddiqui, M.K.; Baig, A.Q. Topological Characterization of the symmetrical structure of Bismuth Tri-Iodide. Symmetry 2018, 10,201.

Young ChelKwun; Abaidur Rehman Virk, On the Multiplicative Degree-Based Topological Indices of SiliconCarbon Si2C3-I[p,q] and Si2C3-II[p,q], J. Math. Symmetry,2018, 10,320.

Haidar Ali; Muhammad Ahsan Binyamin, On the Degree-Based Topological Indices of Some Derived Networks, J. Math. 2019, 70612.

Bondy. J. A.; Murthy, U.S.R. Graph Theory With Applications; Macmillan: London, UK,1976, Volume 290.

Haidar Ali; FarzanaKousar, On Topological Properties of Planar Octahedron Networks, arXiv: 1910.10296V1, 2019. 